\title{
Gluten sensitivity enteropathy in patients with recurrent aphthous stomatitis
}

\author{
Ramin Shakeri $^{1}$, Farhad Zamani ${ }^{\dagger 2}$, Rasoul Sotoudehmanesh ${ }^{\dagger 1}$, \\ Afsaneh Amiri ${ }^{\dagger 2}$, Mehdi Mohamadnejad ${ }^{\dagger 1,2}$, Fereydoun Davatchi ${ }^{\dagger 3}$, \\ Ali Mohammadi Karakani ${ }^{4}$, Reza Malekzadeh ${ }^{\dagger 1}$ and Farhad Shahram* ${ }^{*} 3$
}

Address: ${ }^{1}$ Digestive Disease Research Center (DDRC), Tehran University of medical sciences, Tehran, Iran, ${ }^{2}$ Gastrointestinal and Liver Disease Research Center (GILDRC), Iran University of Medical sciences, Tehran, Iran, ${ }^{3}$ Rheumatology Research center, Tehran University of medical sciences, Tehran, Iran and ${ }^{4}$ Alborz Hospital, Social Security Organization, Karaj, Iran

Email: Ramin Shakeri - rshakeri@gmail.com; Farhad Zamani - zamani.farhad@gmail.com; Rasoul Sotoudehmanesh - Rsotoudeh@yahoo.com; Afsaneh Amiri - amiri.afsaneh@yahoo.com; Mehdi Mohamadnejad - mohamadnejad@hotmail.com;

Fereydoun Davatchi - davacomtchi@yahoo.com; Ali Mohammadi Karakani - akmohammadi@mail.com; Reza Malekzadeh - malek@ams.ac.ir; Farhad Shahram* - shahram@neda.net

* Corresponding author †Equal contributors

Published: 17 June 2009

BMC Gastroenterology 2009, 9:44 doi:10.1 I86/147|-230X-9-44
Received: 18 September 2008

Accepted: 17 June 2009

This article is available from: http://www.biomedcentral.com/I47I-230X/9/44

(C) 2009 Shakeri et al; licensee BioMed Central Ltd.

This is an Open Access article distributed under the terms of the Creative Commons Attribution License (http://creativecommons.org/licenses/by/2.0), which permits unrestricted use, distribution, and reproduction in any medium, provided the original work is properly cited.

\begin{abstract}
Background: Gluten sensitive enteropathy (GSE) is an autoimmune enteropathy triggered by the ingestion of gluten-containing grains in susceptible individuals. Recurrent aphthous stomatitis (RAS) may be the sole manifestation of GSE. The aim of this study was to determine the prevalence of gluten sensitivity enteropathy (GSE) in a large group of patients with RAS and assess the efficacy of gluten free diet (GFD) on the improvement of aphthous lesions in those who were diagnosed with GSE.
\end{abstract}

Methods: Two hundred and forty seven patients with RAS were included. The patients had at least three aphthous attacks per year. Patients were screened by IgA anti-endomysial antibody (EMA), IgA anti tissue transglutaminase (TTG) and serum IgA level. Those with a positive serology underwent endoscopic biopsies of the duodenal mucosa and patients with negative serology were excluded. The diagnosis of GSE was based on a positive serological test and abnormal duodenal histology. For patients with GSE, gluten free diet was recommended.

Results: Six out of 247 RAS patients had positive TTG test alone, and one had positive EMA and TTG. All 7 patients with positive serologic tests underwent duodenal biopsies. Histological findings were compatible with GSE in all of them (Marsh I in four patients, Marsh II in two patients and Marsh IIIB in one another.). The mean age of GSE patients was $27.42 \pm 10.56$ (range, 13 to 40 ) years old. They were suffering from RAS for an average duration of 4.5 years. All of the 7 GSE patients had not responded to the routine anti-aphthae medications, including topical corticosteroids, tetracycline and colchicine. Four patients who adhered to a strict gluten-free diet showed noticeable improvement in their aphthous lesions over a period of 6 months.

Conclusion: A significant minority (e.g. 2.83\%) of RAS patients have GSE. This could be compared with the $0.9 \%$ prevalence of GSE in the general population of Iran. This study suggests that evaluation for celiac disease is appropriate in patients with RAS. Additionally, the unresponsiveness to conventional anti-aphthae treatment could be an additional risk indicator. 


\section{Background}

Gluten sensitive enteropathy (GSE) is an autoimmune enteropathy triggered by the ingestion of gluten-containing grains in susceptible individuals. The presentations of GSE vary clinically, from atypical (without gastrointestinal symptoms), silent and latent to severe forms with gastrointestinal and neurological complications. Although GSE had been identified mainly in individuals of European descent [1], recent data suggests that celiac disease is a common disorder, not only in populations of European ancestry, but also in developing areas, such as North Africa, Middle East and India [2].

Over the past decades, our knowledge on GSE has improved, and many silent or latent cases of GSE have been diagnosed by screening with serological tests[3,4]. Early diagnosis of GSE allows for immediate treatment with a gluten free diet, restores health, and might prevent the development of potential complications associated with GSE (e.g. non-Hodgkin's lymphoma of the gut). [5,6].

Recurrent aphthous stomatitis (RAS) is one of the most common mucosal diseases Aphthae can affect either gender at any age, although a higher prevalence is noted among children, adolescents and females [1]. The prevalence of RAS in general population is estimated to be at least $5 \%$ [7]. It is one of the important causes of outpatient visits. It has been reported that in 5\% of GSE patients, RAS may be the sole manifestation of the disease [8].

The association between CD and RAS has been evaluated in several studies but conflicting results have been reported [9-11]. Therefore, we conducted this study to determine the frequency of gluten sensitivity enteropathy (GSE) in patients with RAS, using relevant serologic as well as histologic tests. We also aimed to assess the efficacy of gluten free diet (GFD) on the improvement of aphthous lesions in those who were diagnosed with GSE

\section{Methods}

Over a period of 24 months, all patients with RAS who attended the Behcet's clinic of Shariati hospital in Tehran were asked to participate in a screening program for GSE. Patients included in the study had at least three episodes of oral aphthae during the year; exclusion criteria were Behcet's disease, inflammatory bowel disease, systemic lupus erythematousis, tumors of oral cavity, Reiter syndrome and oral lesions due to drugs and radiation. Soft tissues examination was carried out with conventional dental chairs, artificial light, flat mirrors, monouse probe and sterile gauzes. We registered, lesions as RAS if they match one of these three conditions: clinically confirmed by physician, referred by patients themselves and reported by hospital clinical records.
The objectives of the study, as well as the possible necessity for a small bowel biopsy, were explained to the patients. Of 290 eligible patients, 247 agreed to participate in our study. Written informed consent was obtained from each participant, an interviewer completed a clinical questionnaire, and five-milliliter venous blood sample was obtained from each patient for serological investigation of GSE.

The study was performed according to the principles of the Digestive Disease Research Center of Tehran University of Medical Science, and was approved by the Ethics Committee of this center and Rheumatology research center of Tehran University of Medical Science.

A complete history of oral aphthous was taken from all subjects regarding number of aphthous ulcers, duration, size, precipitating factor, response to conventional therapy (such as topical corticosteroids, tetracycline and colchicine) and number of attacks per year.

\section{IgA-Anti endomysial antibody}

Serum IgA antibodies against endomysium were measured by the indirect immunofluorescence method using commercial kits (Biosystem Madrid, Spain). Briefly, patient serum is incubated on tissue sections of monkey esophagus to allow binding of antibodies to the substrate. Any antibodies not bound are removed by rinsing. Bound antibodies of the IgA class are detected by incubation of the substrate with fluorescein-labeled, anti-human immunoglobulin conjugate. Reactions are observed under a fluorescence microscope equipped with appropriate filters. The presence of EMA is demonstrated by an apple green fluorescence of the endomysial lining of smooth muscle bundles. The titer (the reciprocal of the highest dilution giving a positive reaction) of the antibody is then determined by testing serial dilutions.

\section{IgA Tissue transglutaminase antibody}

IgA antibodies to tissue transglutaminase were assessed by ELISA using recombinant human tTG as the antigen (Genesis Diagnostics, Cambridgeshire, UK). Serum samples were diluted to $1: 100$ with distilled water, incubated with antigen for $30 \mathrm{~min}$ at room temperature, washed three times, and subsequently incubated for another $30 \mathrm{~min}$ with antihuman IgA. Optical density was read at $450 \mathrm{~nm}$. Results were expressed in arbitrary units (AU) according to the reference calibrator. The cutoff value for a positive outcome was considered to be $7 \mathrm{AU}$, according to the instruction on the kit.

Serum IgA level was measured to rule out IgA deficiency.

\section{Hematology and biochemistry}

Complementary hematologic and biochemical investigations including complete blood count, platelet, erythro- 
cyte sedimentation rate, C-reactive protein, serum sodium, serum potassium, serum calcium, serum phosphorous, blood urea nitrogen, creatinine, cholesterol, triglyceride, alanine aminotransferase (ALT), aspartate aminotransferase (AST), alkaline phosphatase, total iron binding capacity, ferritin, serum iron, stool guaiac exam (OB), total protein and albumin were performed in patients who had been diagnosed as GSE.

\section{Duodenal biopsy}

Patients with positive serologic test (EMA or tTG) underwent upper endoscopy and four biopsies were taken from the second portion of the duodenum.

Each biopsy was oriented over a filter paper as well as being prepared for standard histological assessment. The histological assessment included examination for significant alteration in main mucosal measurements and lymphocytic infiltration, Based on Marsh criteria [12]:

0: Normal mucosal structure without a significant lymphocytic infiltration

: Lymphocytic enteritis (more than 30 lymphocytes/100 epithelial cells).

: Lymphocytic enteritis and crypt hyperplasia.

ША: Partial villous atrophy.

ШB: Subtotal villous atrophy.

ШC: Total villous atrophy.

\section{Gluten-sensitivity enteropathy diagnosis}

The presence of positive tTG or EMA plus abnormal duodenal histology (Marsh, or Ш) was defined as GSE.

\section{Statistical analysis}

Data are presented as Mean \pm SD or percentage. Statistical analyses were performed using SPSS software version 15.

\section{Results}

One hundred thirty one (e.g. 53\%) out of 247 RAS patients were men. Mean age was $33 \pm 11$ years old (range, 11 to 66 year old). After serologic studies for GSE, one patient had positive EMA and tTG tests and six patients were only tTG positive (four men and three women). All patients underwent upper GI endoscopy and duodenal biopsies. In two patients endoscopic finding was compatible with GSE (mild villous atrophy) and five patients had normal endoscopic view. Pathological finding was compatible with Marsh I in four patients, Marsh II in two patients and Marsh IIIB in one.

The mean age of patients with GSE was $27 \pm 10$ (from 13 to 40 ) with a female/male ratio of $3 / 4$. They were suffering from RAS for an average duration of 4.5 years. All the GSE patients were unresponsive to previous trials of conventional anti-aphthae drugs (e.g. topical corticosteroids, tetracycline and colchicine) (Table 1). Complementary laboratory tests of these patients were normal except mild anemia in two patients. None of them complained from other typical or atypical signs and symptoms of CD.

Four out of the seven GSE started a strict gluten free diet. One of them had Marsh I, two had Marsh II, and one had Marsh IIIB lesion. All of them showed a significant improvement within 2-6 months after beginning of GFD.

Table I: Characteristics of subjects with positive serologic antibodies for gluten-sensitive enteropathy

\begin{tabular}{|c|c|c|c|c|c|c|c|c|c|}
\hline Patient no. & sex & age & $\begin{array}{l}\text { Duration of } \\
\operatorname{RAS}(y)\end{array}$ & $\begin{array}{l}\text { No. of attacks } \\
\text { per year }\end{array}$ & $\begin{array}{l}\text { Response to } \\
\text { conventional } \\
\text { therapy }\end{array}$ & serology & $\begin{array}{l}\text { Marsh } \\
\text { classification }\end{array}$ & $\begin{array}{l}\text { Other } \\
\text { symptoms }\end{array}$ & $\begin{array}{l}\text { Improvement } \\
\text { with GFD }\end{array}$ \\
\hline 1 & $\mathrm{~F}$ & 13 & 2 & $12<$ & No & tTG + & II & Anemia & Yes \\
\hline 2 & $M$ & 33 & 5 & $12<$ & No & tTG + & 1 & No & -- \\
\hline 3 & $\mathrm{~F}$ & 40 & 7 & 6 to 12 & No & tTG + & 1 & No & --- \\
\hline 4 & $M$ & 40 & 6 & 6 to 12 & No & tTG + & 1 & No & --- \\
\hline 5 & M & 18 & 3 & $12<$ & No & $\mathrm{AEA}+\& \mathrm{tTG}+$ & III & Anemia & Yes \\
\hline 6 & $M$ & 25 & 5 & $12<$ & No & tTG + & 1 & No & Yes \\
\hline 7 & $\mathrm{~F}$ & 23 & 4 & 6 to 12 & No & $\mathrm{tTG}+$ & II & No & Yes \\
\hline
\end{tabular}

tTG: tissue transglutaminase antibody, EMA: anti endomysial antibody. 
In the four patients, the mean number of attacks of the aphthous lesions was $6.25 \pm 0.96$ in the last 6 months proceeding to GFD and decreased to $1.5 \pm 0.58$ over the first 6 months of GFD adherence.

\section{Discussion}

In this study of a large group of patients with RAS, a $2.83 \%$ prevalence of GSE was observed, as compared with an estimated prevalence of $0.9 \%$ in the general population of Iran [13].

In order to avoid the controversy in the definition of CD, we used the term "gluten sensitive enteropathy" rather than celiac disease to describe patients with any degrees of intestinal damage together with positive serologic tests.

It has been reported that RAS is at least among the fifth commonest presentations of CD [14-16]. Furthermore, oral mucosal lesions or dental enamel defects may be the sole presenting features of celiac disease [17].

Despite detailed investigations, RAS still has an unknown etiology and poorly effective management [18,19]. Genetic, immunological and microbial factors may play a role in the pathogenesis of RAS, whereas attacks may be precipitated by local trauma, stress, food intake, some drugs, hormonal changes or vitamin and trace element deficiencies [19]. RAS can arise in some systemic disorders including: Behcet's disease $[20,21]$, Sweet's syndrome $[22,23]$, MAGIC syndrome [24], inflammatory bowel disease $[25,26]$ and gluten-sensitivity enteropathy (celiac disease) [27].

The association between RAS and gluten-sensitivity enteropathy (GSE) was proposed in 1976 by Ferguson et al [28] when they found $24 \%$ of patients with RAS showed histological evidence of CD on jejunal biopsy. Nevertheless, there is still considerable dispute concerning the actual prevalence of CD among patients with RAS, as different studies have reported different prevalence of CD in RAS patients [29-34]. On the other hand in recent years, some articles are published which expressed little or no significant etiological link between RAS and CD, and added that screening RAS patients for key serological markers of CD is of little clinical value $[10,1]$. Currently, there is no approved recommendation which can be used by clinicians to approach patients with RAS regarding celiac disease. Comparing to the previous studies, this study with a large group of patients and by using two more sensitive and specific serologic tests plus duodenal biopsy helped us reach a reliable conclusion. Prevalence of GSE in patients with RAS was $2.83 \%$, which is about 3 -fold higher than that expected in general population of Iran $(0.9 \%)$ [13].
The effect of gluten-free diet (GFD) on remission of RAS is still uncertain, as dietary withdrawal of gluten occasionally results in significant benefit whereas some studies reported it ineffective $[11,35,36]$. Four patients accepted to start GFD, and all of them showed a significant improvement within 2-6 months after beginning of GFD. Furthermore anemia resolved after 6 months of follow up in the two patients who suffered from anemia.

Many physicians may still consider the gastrointestinal signs and symptoms as a main manifestation of celiac patients whereas recent studies demonstrated that gastrointestinal presentations may be absent in GSE patients especially in the beginning of the disease. In this study, none of our GSE patients had any gastrointestinal symptoms. Therefore, gastrointestinal symptoms are sometimes absent in the setting of the disease and RAS could be the first or the sole presentation of GSE.

Our study has some limitations. We did not take duodenal biopsies from the patients who had negative serological tests. It has been reported that the sensitivities of the serological tests are decreased in GSE patients with minor mucosal damages [37,38]. We cannot exclude the possibility of missing some GSE patients with negative serological tests and Marsh I/II mucosal lesions (e.g. seronegative GSE). However, a patient with negative serological test and duodenal mucosal lesion may suffer from other disorders like autoimmune enteropathy, giardiasis, common variable immunodeficiency, tropical sprue, peptic duodenitis, Crohn's disease etc. Including such patients (e.g. those with negative serological tests with duodenal mucosal damage) in the spectrum of GSE could increase the rate of false positive results; unless symptomatic and histological improvements are confirmed by gluten free diet. Therefore, in the epidemiological studies, a positive result from a highly specific serological test (e.g. EMA, or tTG) together with any degree of duodenal mucosal lesion provide reasonable criteria for identifying patients with GSE.

\section{Conclusion}

In conclusion, a subset of RAS patients suffers from GSE. GSE should be considered in RAS patients; unresponsiveness to conventional anti-aphthae treatment could be an additional risk indicator. Implementation of GFD may prevent the complications of GSE and effectively treat RAS.

\section{Competing interests}

The authors declare that they have no competing interests.

\section{Authors' contributions}

RS Participated in Conception, design, undertaking study and manuscript writing. FZ Participated in Design, under- 
taking study and manuscript writing. R S: Participated in Conception and experimental design. AA Participated in Undertaking study, data analysis, manuscript writing. MM Participated in Conception, design, data analysis, manuscript writing. FD Participated in Conception and experimental design. AMK Participated in undertaking study, laboratory works. RM Participated in Conception and experimental design, manuscript writing. FS Participated in Conception and experimental design, manuscript writing. All authors read and approved the final manuscript.

\section{Acknowledgements}

We kindly thank Dr. E. Scott Swenson from Yale medical school for his valuable comments. We also thank the staff of GILDRC, DDRC and Department of Pathology of Shariati Hospital for their assistance.

\section{References}

I. Sedghizadeh PP, Shuler CF, Allen CM, Beck FM, Kalmar JR: Celiac disease and recurrent aphthous stomatitis: a report and review of the literature. Oral Surg Oral Med Oral Pathol Oral Radiol Endod 2002, 94:474-8.

2. Catassi C: [The global village of celiac disease]. Recenti Prog Med 200I, 92:446-50.

3. Biagi F, Corazza GR: Clinical features of coeliac disease. Dig Liver Dis 2002, 34:225-8.

4. Martucci S, Biagi F, Di Sabatino A, Corazza GR: Coeliac disease. DigLiver Dis 2002, 34:SI50-3.

5. Somech R, Spirer : Celiac disease: extraintestinal manifestations, associated diseases, and complications. Adv Pediatr 2002, 49:19I-20I.

6. Green PH, Fleischauer AT, Bhagat G, Goyal R, Jabri B, Neugut Al: Risk of malignancy in patients with celiac disease. Am J Med 2003, II5:191-5.

7. Neville BW, DDACBJE: Oral and maxillofacial pathology. 2nd edition. Philadelphia: WB Saunders; 2002.

8. Tursi A, Giorgetti G, Brandimarte G, Rubino E, Lombardi D, Gasbarrini G: Prevalence and clinical presentation of subclinical/ silent celiac disease in adults: an analysis on a 12 -year observation. Hepatogastroenterology 200I, 48:462-4.

9. Nowak M, Dziechciarz P, Dwilewicz-Trojaczek J: The frequency of coeliac disease occurrence in patients with recurrent aphthous stomatitis (RAS)-preliminary report. Wiad Lek 2002, 55:542-6.

10. Robinson NA, Porter SR: Low frequency of anti-endomysial antibodies in recurrent aphthous stomatitis. Ann Acad Med Singapore 2004, 33:43-7.

II. Tyldesley WR: Recurrent oral ulceration and coeliac disease. A review. Br Dent J 1981, I5 I:81-3.

12. Marsh MN: The natural history of gluten sensitivity: defining, refining and redefining. $Q J M$ 1995, 88:9-13.

13. Akbari MR, Mohammadkhani A, Fakheri H, Javad ZM, Shahbazkhani B, Nouraie M, Sotoudeh M, Shakeri R, Malekzadeh R: Screening of the adult population in Iran for celiac disease:comparison of the tissue-transglutaminase antibody andanti-endomysial antibody tests. Eur J Gastroenterol Hepatol 2006, I8: I I8I-6.

14. Cilleruelo Pascual ML Román Riechmann E, Jiménez Jiménez J, Rivero Martín MJ, Barrio Torres J, Castaño Pascual A, Campelo Moreno O, Fernández Rincón A: Silent celiac disease: exploring the iceberg in the school-aged population. An Esp Pediatr 2002, 57:321-6.

15. de Freitas IN, Sipahi AM, Damião AO, de Brito T, Cançado EL, Leser PG, Laudanna AA: Celiac disease in Brazilian adults. J Clin Gastroenterol 2002, 34:430-4.

16. Dobru D, Pascu O, Tană M, Gheorghe C, Goldiş A, Bălan G, Olteanu I, Fraticiu A, Dumitru E, Miuescu E, Mulder C, Podoleanu S: The prevalence of coeliac disease at endoscopy units in Romania: routine biopsies during gastroscopy are mandatory (a multicentre study). Rom / Gastroenterol 2003, 12:97-100.

17. Lähteenoja $H$, Irjala $K$, Viander $M$, Vainio $E$, Toivanen $A$, Syrjänen $S$ : Oral mucosa is frequently affected in patients with dermatitis herpetiformis. Arch Dermatol 1998, 134:756-8.
18. Veller-Fornasa C, Bezze G, Rosin S, Lazzaro M, Tarantello M, Cipriani R: Recurrent aphthous stomatitis and atopy. Acta Derm Venereol 2003, 83:469-70.

19. Natah SS, Konttinen YT, Enattah NS, Ashammakhi N, Sharkey KA, Häyrinen-Immonen R: Recurrent aphthous ulcers today: a review of the growing knowledge. Int J Oral Maxillofac Surg 2004, 33:22I-34.

20. Mittal R, Chopra A, Handa F: Sutton's aphthae and Behçet's syndrome. Indian J Dermatol 1985, 30: I7-21.

21. Jankowski J, Crombie I, Jankowski R: Behçet's syndrome in Scotland. Postgrad Med J 1992, 8:566-70.

22. Driesch $P$ Von den: Sweet's syndrome (acute febrile neutrophilic dermatosis). J Am Acad Dermatol 1994, 31:535-56.

23. Driesch $P$ Von den, Gomez RS, Kiesewetter F, Hornstein OP: Sweet's syndrome: clinical spectrum and associated conditions. Cutis 1989, 44:193-200.

24. Orme RL, Nordlund J], Barich L, Brown T: The MAGIC syndrome (mouth and genital ulcers with inflamed cartilage). Arch Dermatol 1990, 1 26:940-4.

25. Kochhar R, Mehta SK, Nagi B, Bhatia V, Goenka MK, Malik AK Extraintestinal manifestations of idiopathic ulcerative colitis. Indian J Gastroenterol 1991, 10:88-9.

26. Ghandour K, Issa M: Oral Crohn's disease with late intestinal manifestations. Oral Surg Oral Med Oral Pathol I99I, 72:565-7.

27. Ferguson R, Basu MJ, Asquith P, Cooke WT: Proceedings: Recurrent aphthous ulceration and its association with coeliac disease. Gut 1975, 16:393.

28. Ferguson R, Basu MK, Asquith P, Cooke WT: Jejunal mucosal abnormalities in patients with recurrent aphthous ulceration. $\mathrm{Br}$ Med J 1976, I: I I-I3.

29. Wray D, Ferguson MM, Mason DK, Hutcheon AW, Dagg JH: Recurrent aphthae: treatment with vitamin $B / 2$, folic acid, and iron. $\mathrm{Br}$ Med J 1975, 2:490-3.

30. Rose JD, Smith DM, Allan FG, Sircus W: Recurrent aphthous ulceration and jejunal biopsy. Br Med J 1978, I:I I45.

31. Ferguson MM, Wray D, Carmichael HA, Russell RI, Lee FD: Coeliac disease associated with recurrent aphthae. Gut 1980, 2I:223-6.

32. Tyldesley WR: Recurrent oral ulceration and coeliac disease. A review. Br Dent J 198I, I5I:8I-3.

33. Merchant NE, Ferguson MM, Ali A, Hole DJ, Gillis CR: The detection of IgA-reticulin antibodies and their incidence in patients with recurrent aphthae. J Oral Med 1986, 4I:3 I-4.

34. Veloso FT, Saleiro JV: Small-bowel changes in recurrent ulceration of the mouth. Hepatogastroenterology 1987, 34:36-7.

35. Wray D: Gluten-sensitive recurrent aphthous stomatitis. Dig Dis Sci 198I, 26:737-40.

36. O'Farrelly C, O'Mahony C, Graeme-Cook F, Feighery C, McCartan $\mathrm{BE}$, Weir DG: Gliadin antibodies identify gluten-sensitive oral ulceration in the absence of villous atrophy. J Oral Pathol Med 1991, 20:476-8.

37. Rostami K, Kerckhaert J, Tiemessen R, von Blomberg BM, Meijer JW, Mulder C): Sensitivity of antiendomysium and antigliadin antibodies in untreated celiac disease: disappointing in clinical practice. Am J Gastroenterol 1999, 94:888-94.

38. Tursi A, Brandimarte G, Giorgetti GM: Prevalence of antitissue transglutaminase antibodies in different degrees of intestinal damage in celiac disease. J Clin Gastroenterol 2003, 36:2 19-21.

\section{Pre-publication history}

The pre-publication history for this paper can be accessed here:

http://www.biomedcentral.com/1471-230X/9/44/pre pub 